\title{
FORMACIÓN EN COMPETENCIAS DEL ALUMNADO DE EDUCACIÓN EN LA ATENCIÓN A LA DIVERSIDAD: DIFERENCIAS SEGÚN LA TITULACIÓN
}

\author{
Carolina Fernández Jiménez \\ Facultad de Ciencias de la Educación \\ Universidad de Granada \\ carolina@ugr.es \\ Mirian Hervás Torres \\ Facultad de Ciencias de la Educación \\ Universidad de Granada \\ Marta Aparicio Puerta \\ Facultad de Ciencias de la Educación \\ Universidad de Granada \\ M. Tamara Polo Sánchez \\ Facultad de Ciencias de la Educación \\ Universidad de Granada \\ Susana Tallón Rosales \\ Facultad de Ciencias de la Educación \\ Universidad de Granada
}

Fecha de Recepción: 19 Febrero 2019

Fecha de Admisión: 30 Abril 2019

\section{RESUMEN}

La Educación Superior defiende actualmente un nuevo paradigma educativo, que incluya metodologías activas y estrategias de evaluación que se centren en el desarrollo de competencias como medio para lograr la educación integral de los estudiantes. El presente estudio describe la aplicación de una de esas metodologías activas, el aprendizaje basado en problemas (ABP), dirigido a alumnado de educación que cursa materias relacionadas con la atención a la diversidad en diferentes titulaciones. La muestra estaba formada por 239 estudiantes de la Facultad de Ciencias de la Educación de la Universidad de Granada. Los resultados del estudio muestran diferencias en el logro de competencias según la titulación cursada.

Palabras clave: diversidad; aprendizaje basado en problemas; competencias; formación

\section{ABSTRACT}

Training in competences of the pupils of education in the attention to the diversity: differences according to the degree. The Higher Education defends for a new educational paradigm, 


\section{FORMACIÓN EN COMPETENCIAS DEL ALUMNADO DE EDUCACIÓN EN LA ATENCIÓN A LA DIVERSIDAD: DIFERENCIAS SEGÚN LA TITULACIÓN}

one involving active methodologies and evaluation strategies that focus on development of basic competencies as the means to achieve the comprehensive education of students. This paper describes a study based on the application of one of these active methodologies, Problem-Based Learning (PBL), for Education students enrolled in courses that address diversity awareness in different degrees. The sample consisted of 239 students of the Faculty of Sciences of the Education of the University of Granada. The results show differences in the achievement of competences according to the degree.

Keywords: diversity; problem-based learning; competences; education training

\section{INTRODUCCIÓN}

La formación universitaria de los futuros profesionales de la educación ha de adecuarse a un nuevo perfil profesional que responda a la diversidad de personas y contextos. Como temática transversal, la formación en la atención a la diversidad debe perseguir el desarrollo en el alumnado de ciertas competencias que generalmente se relacionan con la resolución de problemas de forma multidisciplinar y colaborativa, siendo efectivo para ello el uso de las denominadas metodologías activas (Palomares, 2011). Este aprendizaje basado en competencias está asociado a la construcción de los nuevos estudios de grado en torno a un conjunto de competencias profesionales relacionadas con el ejercicio de la profesión que el futuro graduado desarrollará. El diseño de las asignaturas debe basarse en competencias y objetivos de aprendizaje tal y como se plantea desde el EEES (Ruiz, 2004), lo cual conlleva una importante renovación de las metodologías docentes. Este nuevo modelo exige el empleo de metodologías activas, nuevas estrategias didácticas y evaluación por competencias que requieren de la interacción con el alumnado, el trabajo en grupo y una evaluación ligada a las tareas de clase. En este contexto, el aprendizaje basado en problemas (ABP) se constituye como una herramienta útil que encaja perfectamente en las nuevas metodologías (Mateos, Eguzkitza y González, 2012). En el enfoque del ABP se fomenta la autonomía cognoscitiva, se enseña y se aprende a partir de problemas que tienen significado para los estudiantes, se utiliza el error como una oportunidad más para aprender y no para castigar y se le otorga una valiosa importancia a la autoevaluación. La autoevaluación consiste en la evaluación que lleva a cabo el alumno del propio aprendizaje, así como de los factores relacionados con éste, siendo la autoevaluación un objetivo de aprendizaje en sí mismo (Villardón, 2006).

Cuando es realizada por los propios estudiantes la autoevaluación parece incrementar el aprendizaje al posibilitar la detección de sus puntos fuertes y débiles en este proceso (Gimeno y Gallego, 2007). Como en cualquier otro método de evaluación, la elección de los instrumentos a utilizar para la recogida de información resulta fundamental. Las rúbricas o matrices de evaluación constituyen un ejemplo de las herramientas disponibles para que el profesorado pueda llevar a cabo una evaIuación formativa (Conde y Pozuelo, 2007). Su diseño y aplicación permiten concretar los criterios con los que valorar las competencias y el aprendizaje, exponiendo al alumnado las expectativas de logro del profesorado respecto a su desempeño (Etxabe, Aranguren y Losada, 2011). Mediante las rúbricas el estudiante puede estimar su propia calificación, así como el nivel de competencias adquirido lo que aumenta la motivación y responsabilidad del alumnado sobre su propio proceso de aprendizaje (Villalustre y Del Moral, 2010). Los positivos efectos que estos instrumentos pueden provocar cuando se emplean en la autoevaluación justifican su uso sistemático en el contexto universitario (Rodríguez, Ibarra, Gallego, Gómez y Quesada, 2012).

En el presente estudio se pretende comprobar si existen diferencias en la percepción del alumnado sobre la adquisición de competencias vinculadas a la atención a la diversidad según la titulación cursada, utilizando como metodología docente el aprendizaje basado en problemas. 


\section{MÉTODO}

\section{Muestra}

La presente investigación se ha llevado a cabo con alumnado de $2^{\circ}$ curso en tres materias que abordaban la atención a la diversidad en diferentes titulaciones impartidas en la Facultad de Ciencias de la Educación, de la Universidad de Granada. Participaron 229 estudiantes (33 hombres y 196 mujeres) con una media de edad de 22,15 años y desviación típica de 3,760 (Tabla 1).

Tabla 1

Titulaciones, materias implicadas y número de alumnado

\begin{tabular}{|c|c|c|c|c|}
\hline & $\begin{array}{c}\text { Educación Social } \\
\text { Materia: } \\
\text { Capacidades } \\
\text { Diversas y } \\
\text { Educación Social }\end{array}$ & $\begin{array}{c}\text { Educación } \\
\text { Infantil } \\
\text { Materia: } \\
\text { Trastornos del } \\
\text { Desarrollo }\end{array}$ & $\begin{array}{c}\text { Pedagogía } \\
\text { Materia: } \\
\text { Procesos y Técnicas de } \\
\text { Intervención } \\
\text { Psicoeducativa }\end{array}$ & Totales \\
\hline Hombres & 11 & 15 & 7 & 33 \\
\hline Mujeres & 44 & 126 & 26 & 196 \\
\hline$N$ & 55 & 141 & 33 & 229 \\
\hline$\%$ & 24 & 61,7 & 14,3 & 100 \\
\hline
\end{tabular}

\section{Procedimiento e Instrumentos}

Se tomaron como referencia las competencias recogidas en los Libros Blancos para los Títulos de Grado de Educación social, Pedagogía (Aneca, 2005) y Magisterio (Aneca, 2004) y más concretamente aquéllas consideradas por titulados y asociaciones, más relevantes para el perfil de atención a la diversidad (Aneca, 2004; 2005). Se eligieron a) la capacidad de organización y planificación, competencia que se tiene muy en cuenta a nivel profesional y en las organizaciones, pero poco considerada en la formación universitaria (Marzo, Pedraja y Rivera, 2006); b) la comunicación oral y escrita, habilidades básicas para los profesionales de la educación (Fernández, Torío y Viñuela, 2008), escasamente desarrolladas en los estudiantes universitarios (Arráez, et al., 2008; LópezJusticia, Hernández, Fernández, Polo y Chacón, 2008); c) el trabajo en equipo, d) las habilidades interpersonales, competencias consideradas muy relevantes para las organizaciones (Torrelles et al., 2011) y relacionadas con el éxito social y personal aunque escasas en alumnado de educación (Fuentes, González y Raposo, 2008; García de la Vega, 2010; López-Justicia, et al., 2008); y e) el reconocimiento y respeto hacia la diversidad, competencia fundamental para aquellos profesionales que atienden a este colectivo (López, 2009; Martínez, 2011).

Con el fin de conocer el nivel de logro del alumnado en estas competencias al comenzar el curso, en el mes de octubre y previamente a la aplicación del ABP, se realizó una autoevaluación mediante rúbricas. Posteriormente, al finalizar la materia en el mes de enero, se utilizaron nuevamente las rúbricas para una segunda autoevaluación. Se indicó a los estudiantes que el resultado de la autoevaluación se contemplaría en la nota final representando ésta un $5 \%$ de la calificación final.

\section{RESULTADOS}

Para el análisis de los datos se utilizó el paquete estadístico SPSS en su versión 23.

Se realizó un ANOVA que mostraba la existencia de diferencias significativas en ciertos indicadores de las competencias evaluadas en función de la titulación cursada (Tabla 4). En los descriptores referidos a la competencia organización y planificación, se dan diferencias significativas en la 
"implicación en las tareas" ( $F 2,228=10,706, p=, 000)$, y "asistencia a clase" ( $F 2,228=9,459, p=, 000)$, siendo el alumnado de Educación Infantil los que perciben mayor nivel de logro. Los análisis posthoc mediante la prueba de Tukey revelaron diferencias significativas entre la titulación de Educación Social respecto a las de Infantil $(p=, 000)$ y Pedagogía $(p=, 020)$. En la "planificación y organización de las sesiones de trabajo", los estudiantes de Pedagogía consideran haber mejorado más en este aspecto ( $F 2,228=7,624, p=, 001)$. Las comparaciones post-hoc mostraron diferencias nuevamente entre Educación Social y la titulación de Infantil $(p=, 005)$ y Pedagogía $(p=, 001)$. Referida a la competencia trabajo en equipo, se observan diferencias significativas en el "trabajo cooperativo", siendo éste un comportamiento que mejora especialmente para el alumnado de Educación Social ( $F 2,228=5,888, p=, 003)$. Realizadas las pruebas post hoc, se encontraron diferencias al comparar Educación Social y la titulación de Infantil $(p=, 003)$. Respecto a la habilidad para "ponerse en el lugar de la persona con discapacidad" también el alumnado de Educación Social logra mejores resultados ( $F 2,228=19,731, p=, 000)$, siendo significativas las diferencias entre esta titulación y Pedagogía $(p=, 000)$ e Infantil $(p=, 000)$. Resultados similares obtenemos con el descriptor "conocer las dificultades que encuentra este colectivo" ( $F 2,228=16,384, p=, 000)$, mostrando las comparaciones post hoc diferencias significativas entre Educación Social y Pedagogía $(p=, 008)$ e Infantil $(p=, 000)$.

Tabla 4

Resultados ANOVA. Diferencias según titulación

\begin{tabular}{|c|c|c|c|c|c|c|c|c|}
\hline \multicolumn{9}{|c|}{ Titulación } \\
\hline & \multicolumn{2}{|c|}{$\begin{array}{l}\text { Educación } \\
\text { Infantil }\end{array}$} & \multicolumn{2}{|c|}{$\begin{array}{l}\text { Educación } \\
\text { Social }\end{array}$} & \multicolumn{2}{|c|}{ Pedagogía } & \multirow[t]{2}{*}{$\mathrm{F}$} & \multirow[t]{2}{*}{$\mathrm{p}$} \\
\hline & $\mathrm{M}$ & DT & $\mathrm{M}$ & DT & $\mathrm{M}$ & DT & & \\
\hline Implicación tareas & 3,75 & ,46 & 3,38 &, 52 & 3,69 & ,64 & 10,706 &, 000 \\
\hline Asistencia a clase & 3,59 &, 53 & 3,38 & ,49 & 3,16 & ,67 & 9,459 &, 000 \\
\hline Planificación trabajo & 2,79 &, 54 & 2,49 & ,69 & 2,97 & ,64 & 7,624 & ,001 \\
\hline $\begin{array}{l}\text { Trabaja con } \\
\text { compañeros }\end{array}$ & 2,98 & ,79 & 3,13 &, 72 & 2,97 & ,64 & ,826 & ,439 \\
\hline Comparte información & 3,31 & ,71 & 3,36 &, 72 & 3,41 &, 75 & ,282 & ,754 \\
\hline Tolerancia & 3,45 & ,60 & 3,53 & ,60 & 3,38 &, 70 & ,644 &, 526 \\
\hline Trabajo cooperativo & 3,14 &, 84 & 3,55 &, 50 & 3,38 & ,75 & 5,888 & ,003 \\
\hline \multicolumn{9}{|l|}{ Solicitar ideas } \\
\hline $\begin{array}{l}\text { Ponerse lugar persona } \\
\text { con discapacidad }\end{array}$ & 2,85 & ,70 & 3,51 &, 50 & 2,94 & ,71 & 19,731 &, 000 \\
\hline $\begin{array}{l}\text { Soy sensible a las } \\
\text { necesidades de las } \\
\text { personas con } \\
\text { discapacidad }\end{array}$ & 3,32 & ,61 & 3,40 &, 56 & 3,47 &, 50 & 1,058 & ,349 \\
\hline $\begin{array}{l}\text { Conocer dificultades } \\
\text { personas con } \\
\text { discapacidad }\end{array}$ & 2,92 &, 75 & 3,56 &, 58 & 3,09 & ,73 & 16,384 & ,000 \\
\hline
\end{tabular}




\section{DISCUSIÓN}

El estudio descrito propone la autoevaluación como alternativa para valorar la adquisición de competencias relacionadas con la atención a la diversidad por el alumnado de educación empleando el ABP como metodología docente. Finalmente, se comprueba que existen diferencias en la percepción que el alumnado tiene de los indicadores de las competencias que mejoran tras la aplicación del ABP según la titulación. Mientras el alumnado de educación infantil y pedagogía perciben más nivel de logro en comportamientos relacionados con la organización y planificación de las tareas, los estudiantes que cursan educación social creen haber mejorado más en el trabajo cooperativo y especialmente en el reconocimiento de la discapacidad. Es probable que estos resultados respondan al diferente valor que para el alumnado tendrían estas competencias a nivel profesional. En este sentido y como señala el Libro Blanco para el título de Grado de Educación Social (Aneca, 2005) el educador social desarrolla su labor en un contexto multidisciplinar trabajando en equipo. Es factible que el alumnado de esta titulación considere fundamental mejorar en esta habilidad tan importante en su vida profesional. Igual ocurriría con el reconocimiento de la discapacidad, una competencia que sería esencial para los profesionales que trabajan con este colectivo (López, 2009; Martínez, 2011). En el caso de los estudiantes de infantil y pedagogía, posiblemente la organización y planificación sean consideradas competencias más significativas para su futuro profesional y por tanto sea mayor su motivación y esfuerzo en aprender éstas.

\section{REFERENCIAS BIBLIOGRÁFICAS}

Agencia nacional de evaluación de la calidad y acreditación. (2004). Libro Blanco. Título de Grado en Magisterio (Vol. 1). Madrid. Extraído el 20 de noviembre de 2013, de http://www.aneca.es/var/media/150404/libroblanco_jun05 magisterio1.pdf

Agencia nacional de evaluación de la calidad y acreditación. (2005). Libro Blanco. Título de Grado de Pedagogía y Educación Social. Extraído el 20 de noviembre de 2013, de http://www.aneca.es/var/media/150392/libroblanco pedagogia1 0305.pdf

Arráez, L., Millán, J., Carabantes, D., Lozano, R., Iglesias, I., Palacios, E., Del Castillo, B., y Nogales, A. (2008). Adquisición de competencias transversales en alumnos de pregrado de Ciencias de la Salud en la Universidad Complutense: una experiencia positiva. Educación Médica, 11(3), 169-177.

Etxabe, J., Aranguren, K., y Losada, D. (2011). Diseño de rúbricas en la formación inicial de maestros/as. Revista de Formación e Innovación Educativa Universitaria, 4(3), 156-169.

Fernández, C. M., Torío, S. y Viñuela, Mạ. P. (2008). La comunicación oral como competencia transversal de los estudiantes de pedagogía y magisterio: presentación del diseño metodológico de una innovación para su trabajo en el aula. Revista de enseñanza universitaria, 31, 26-38.

Fuentes, E., González, M., y Raposo, M. (2008). Desarrollar competencias en la formación inicial de maestros: valoraciones, retos y propuestas. Innovación educativa, 18, 45-68.

García de la Vega, A. (2010). Aprendizaje basado en problemas: aplicaciones a la didáctica de las Ciencias Sociales en la Formación Superior. CIDd: II Congrés Internacional de Didactiques 2010. Girona: Universitat de Girona

Gimeno, M., y Gallego, S. (2007). La autoevaluación de las competencias básicas del estudiante de Psicología. Revista de Psicodidáctica, 12(1), 7-27.

López, M. (2009). La inclusión educativa de los alumnos con discapacidades graves y permanentes en la Unión Europea. Relieve, 15(1), 1-20. Recuperado de http://www.uv.es/RELIEVE/v15n1/RELIEVEV15n1_5.htm.

López-Justicia, M. L., Hernández, C. M., Fernández, C., Polo, T., y Chacón, H. (2008). Características 
formativas y socioafectivas del alumnado de nuevo ingreso en la Universidad. Revista Electrónica de Investigación Psicoeducativa, 14(6), 95-116.

Martínez, M. J. (2011). Formación de maestros, atención educativa a alumnos con plurideficiencia y estimulación sensoriomotriz. REIFOP, 14(1), 137-150. Recuperado de http//www.aufop.com.

Marzo, M., Pedraja, M., y Rivera, P. (2006). Las competencias profesionales demandadas por las empresas: el caso de los ingenieros. Revista de Educación, 341,643-66.

Mateos, E., Eguzkitza, J. M., y González, J. (2012). Implantación de metodología de Aprendizaje Cooperativo basada en Proyectos. XX Congreso Universitario de Innovación Educativa en las Enseñanzas Técnicas XXCUIEET. Recuperado de http://www.eiic.ulpgc.es/documentoscongresos/Esperanza\%20Mateos.pdf

Palomares, A. (2011). El modelo docente universitario y el uso de nuevas metodologías en la enseñanza, aprendizaje y evaluación. Revista de Educación, 355, 591-604. Recuperado de http://www.revistaeducacion.educacion.es/re355/re355_25.pdf

Rodríguez, G., Ibarra, M., Gallego, B., Gómez, M. A., y Quesada, V. (2012). La voz del estudiante en la evaluación del aprendizaje: un camino por recorrer en la universidad. RELIEVE, 18(2). Recuperado de http://www.uv.es/RELIEVE/v18n1/RELIEVEv18n2 2.htm

Ruiz, M. (2004). El Espacio Europeo de Educación Superior y las titulaciones de Educación. Revista interuniversitaria de formación del profesorado, 18(3), 61-79.

Torrelles, C., Coiduras, J., Isus, S., Carrera, F., París, G., y Cela, J. M. (2011). Competencia de trabajo en equipo: definición y categorización. Revista de Currículum y Formación del profesorado, 15(3), 330-344.

Villalustre, L., y Del Moral, M. E. (2010). E-portafolios y rúbricas de evaluación en rural net. PixelBit: Revista de medios y educación, 37, 93-105.

Villardón, M. L. (2006). Evaluación del aprendizaje para promover el desarrollo de competencias. Educatio siglo XXI, 24. Recuperado de http://revistas.um.es/index.php/educatio/article/view/153 